\title{
Publisher's Note: Dichotomy between the Hole and Electron Behavior in Multiband Superconductor FeSe Probed by Ultrahigh Magnetic Fields [Phys. Rev. Lett. 115, 027006 (2015)]
}

M. D. Watson, T. Yamashita, S. Kasahara, W. Knafo, M. Nardone, J. Béard, F. Hardy, A. McCollam, A. Narayanan, S. F. Blake, T. Wolf, A. A. Haghighirad, C. Meingast, A. J. Schofield, H. v. Löhneysen, Y. Matsuda, A. I. Coldea, and T. Shibauchi (Received 5 November 2015; published 20 November 2015)

DOI: 10.1103/PhysRevLett.115.219902 PACS numbers: 74.70.Xa, 71.18.+y, 74.25.Jb, 74.25.Op, 99.10.Fg

This paper was published online on 10 July 2015 with an incorrect reference. Reference [31] should read as "DOI: 10.5287/bodleian:q524jn76v." The reference has been corrected as of 9 November 2015. The reference is incorrect in the printed version of the journal. 